\title{
Environmental effects on the bright end of the galaxy luminosity function in galaxy clusters
}

\author{
R. Barrena ${ }^{1,2}$, M. Girardi ${ }^{3,4}$, W. Boschin ${ }^{5}$, and F. Mardirossian ${ }^{3,4}$ \\ ${ }^{1}$ Instituto de Astrofísica de Canarias, C/Vía Láctea s/n, 38205 La Laguna (Tenerife), Canary Islands, Spain \\ e-mail: rbarrena@iac.es \\ 2 Departamento de Astrofísica, Universidad de La Laguna, Av. del Astrofísico Francisco Sánchez s/n, 38205 La Laguna (Tenerife), \\ Canary Islands, Spain \\ 3 Dipartimento di Fisica dell' Università degli Studi di Trieste - Sezione di Astronomia, via Tiepolo 11, 34143 Trieste, Italy \\ ${ }^{4}$ INAF - Osservatorio Astronomico di Trieste, via Tiepolo 11, 34143 Trieste, Italy \\ 5 Fundación Galileo Galilei - INAF, Rambla José Ana Fernández Perez 7, 38712 Breña Baja (La Palma), Canary Islands, Spain
}

Received 5 December 2011 / Accepted 17 January 2012

\section{ABSTRACT}

\begin{abstract}
Context. The dependence of the luminosity function (LF) of cluster galaxies on the evolutionary state of the parent cluster is still an open question, in particular concerning the formation/evolution of the brightest cluster galaxies.

Aims. We study the bright part of the LFs of a sample of very unrelaxed clusters ("DARC" clusters showing evidence of major, recent mergers) and compare them to a reference sample of relaxed clusters that span a comparable mass and redshift range.

Methods. Our analysis is based on the SDSS DR7 photometric data of ten massive and X-ray luminous clusters $(0.2 \lesssim z \lesssim 0.3)$, always considering physical radii $\left(R_{200}\right.$ or its fractions). We considered $r^{\prime}$ band LFs and used the color-magnitude diagrams $\left(r^{\prime}-i^{\prime}, r^{\prime}\right)$ to clean our samples to consider red and blue galaxies separately.

Results. We find that DARC and relaxed clusters give similar LF parameters and blue fractions. The two samples differ in their content of bright galaxies BGs, $M_{r^{\prime}}<-22.5$. Relaxed clusters have fewer BGs, in particular when considering the outer cluster region $0.5 R_{200}<R<R_{200}$ (by a factor two). However, the cumulative light in BGs is similar for relaxed and DARC samples.

Conclusions. We conclude that BGs grow in luminosity and decrease in number as the parent clusters grow hierarchically, which agrees with a BG formation by merging with other luminous galaxies.
\end{abstract}

Key words. galaxies: clusters: general - galaxies: luminosity function, mass function - cosmology: observations

\section{Introduction}

The study of the formation and evolution of galaxies is a fundamental avenue of research in the process of understanding astrophysical and cosmological questions. The galaxy luminosity function (LF) - the number of galaxies per unit volume in the luminosity interval $L$ and $L+\mathrm{d} L-$ is one of the most direct observational tests of theories of galaxy formation and evolution.

Clusters of galaxies are ideal systems within which to measure the galaxy LF for many galaxies at the same distance. On the other hand, clusters represent an extreme environment for galaxy evolution, either in situ or through the accretion of galaxies within groups that are situated in the filamentary structure of the hierarchical Universe (e.g., Poggianti et al. 2005).

While introducing the modern form of the LF, the so-called "Schechter function", Schechter (1976) suggested that the cluster LF is universal in shape (with a turnover at $M_{B}^{*}=-20.6+$ $5 \log h_{50}$ and a faint-end slope of $\alpha=-1.25$ ). Much progress has been made since then in looking for possible observational signatures of a non-universality of the cluster LF. That different morphological types are characterized by different LFs (Binggeli et al. 1988), and the well-known morphological segregation respect the local density and thus the clustercentric radius (Dressler 1978; Whitmore 1991) makes the study of the cluster LF more complex. In this context, several studies of LF are concerned with the possible variation of the cluster LF with the local projected galaxy density and/or cluster-centric radius. In particular, there are claims for a radius-dependent steepening of the galaxy LF at faint magnitudes, likely caused by red galaxies (e.g., de Propris 1995; Driver et al. 1998; Popesso et al. 2006; Barkhouse et al. 2007). However, the few cases of LFs based on deep spectroscopy exhibit shallower slopes (e.g., Rines \& Geller 2008, and references therein), suggesting that the field contamination might be the cause of the observed steep LFs based on photometry only. Therefore, the question is still far from a definitive conclusion.

Another question concerns the possible dependence of the LF on global cluster properties. Some studies investigated a few well-studied clusters and/or cluster complexes (e.g., Abell 209 in Mercurio et al. 1996; Coma vs. Abell 1367 in Iglesias-Páramo et al. 2003) while other studies used a broad statistical base. In particular, there are indications in favor of correlations between the LF parameters and the cluster mass or its proxies (e.g., richness, luminosity, galaxy velocity dispersion). For instance, Valotto et al. (1997) find that poorer clusters have a flatter LF. But, when the LF is properly calculated within the cluster physical sizes, given by $R_{200}$ or $R_{500}$, the correlation between the dwarf-to-giant ratio and X-ray (or optical) luminosity disappears (cf. Popesso et al. 2006 with Popesso et al. 2004). This suggests that the radial behavior of the LF should be taken into account to obtain unbiased conclusions. However, even when considering an appropriate cluster physical size, the conclusions are not all consistent. For example, Hilton et al. (2005) found that clusters with lower X-ray luminosity have a brighter $M^{*}$, 
but Barkhouse et al. (2007) found no correlation. Recent studies also attempted to analyze more distant clusters, but again without conclusive results (Gilbank et al. 2008; Rudninick et al. 2009, and discussion therein).

Possible correlations with the presence/absence of a $\mathrm{cD}$ galaxy or with the Bautz-Morgan type (the BM-type classification based on the brightest cluster galaxies, BCGs, Bautz \& Morgan 1970), are, maybe, also more interesting for their direct connection with the cluster evolutionary state. Indeed, BCGs or $\mathrm{cD}$ galaxies are expected to be created i) via the merger of giant galaxies in an early phase during the cluster collapse (Merritt 1984), or ii) in a following phase caused by the dynamical friction acting on late-comer galaxies (e.g., Ostriker \& Tremaine 1975), or iii) via the disruption and cannibalization of many faint galaxies in the cluster cores (López-Cruz et al. 1997). The first two mechanisms might reduce the number of bright galaxies, thus shifting $M^{*}$ to a fainter value and, indeed, Barkhouse et al. (2007) found a weak correlation with the BM-type (but see de Propris et al. 2003, for no correlation). Another consequence can be the increment of the gap between the first and the second brightest galaxies in each cluster and, indeed, this magnitude gap is shown to be wider in clusters without substructure and more narrow in clusters with substructure (Ramella et al. 2007).

López-Cruz et al. (1997) found for the second mechanism that seven rich, massive $\mathrm{cD}$ clusters with symmetric X-ray morphology show a flat faint-end slope while a steeper faint-end slope is detected in poorer clusters but also in a rich binary cluster such as the Coma cluster. Somewhat in agreement, Driver et al. (1998) found that the dwarf-to-giant ratio increases with larger BM-types (i.e., likely less evolved clusters). In this context, Valotto et al. (2004) pointed out the importance of the projection effects in determining the faint-end slope. Indeed, in the large cluster sample analyzed by Valotto et al. it is the X-ray selection and not the cluster domination by central galaxies that determines the flatness of the faint-end slope which suggests that real, compact clusters are less affected by the field contamination. However, although this bias could explain possible differences between rich and poor clusters, the reason for the difference between the seven cD relaxed clusters of López-Cruz et al. (1997) and the Coma cluster is not yet clear.

The remarkable study of de Propris et al. (2003) analyzed 60 clusters searching for a possible dependence of the LF on the dynamical status of the cluster and found that the LF is similar for clusters with and without substructure. Very few detailed works are devoted to studying the LFs of subclumps in individual clusters to find possible evidence of cluster mergers (e.g., Durret et al. 2011).

As reported above, the bright end of the LF is particularly interesting because there is a long debate on the BCG formation. For instance, semianalytical models suggest that BCGs assemble surprisingly late with the stars formed very early in many small galaxies (e.g., $50 \%$ at $z=0.5,80 \%$ at $z=0.3$; see de Lucia $\&$ Blaizot 2007). However, photometric and chemical observables suggest that it is difficult to explain giant elliptical by a pure sequence of multiple minor dry mergers or via major dry mergers (e.g., Stott et al. 2010; Ascaso et al. 2011), and numerical simulations are continuously improved to reproduce them better (e.g., Ruszkowski \& Springel 2009). Moreover, galaxy-galaxy mergers are most efficient within small halos with a low velocity dispersion, and the merger of BCGs in clusters requires much time (Dubinski 1998). However, the correlation between BCG luminosity and parent cluster mass strongly suggest that BCGs co-evolves with the cluster (Lin \& Mohr 2004).
In this context, we study the LFs of a sample of massive and very unrelaxed clusters and compare them to a reference sample of relaxed clusters that span a comparable mass and redshift range.

Spectroscopic data for unrelaxed clusters were acquired at the TNG in a long term project (DARC - Dynamical Analysis of Radio Clusters $^{1}$ - e.g., Girardi et al. 2007) to study clusters exhibiting large, diffuse radio sources, i.e. radio halos and relics. These radio sources are rare phenomena and are caused by the synchrotron radio emission of widespread relativistic particles embedded in the cluster magnetic field (e.g., see Feretti et al. 2002 , for a review). Cluster mergers have been proposed to provide the large amount of energy necessary for electron reacceleration to relativistic energies and for magnetic field amplification (e.g., Tribble 1993; Ensslin et al. 1998; Brunetti et al. 2009) or were thought to explain the time-dependence of the magnetic fields in recent models based on secondary electrons (Keshet \& Loeb 2010; Keshet 2011). Indeed, from the observational point of view, there is growing evidence of the connection between diffuse radio emission and cluster merging, given that up to now diffuse radio sources have been detected only in merging systems. In most past studies the cluster dynamical state was derived from X-ray observations (see Buote 2002; Feretti 2006, 2008; Cassano et al. 2010). Our analyses, which are based on optical data and the kinematics of member galaxies, also detect strong subclustering and find evidence of cluster mergers. Accordingly, clusters with radio halos/relics are ideal cases for studying the effect of the cluster formation on the LF.

Here we analyze a subsample of five DARC clusters that we analyzed in the past, which all have available photometry in the the Sloan Digital Sky Survey (SDSS). The DARC clusters form our sample of unrelaxed clusters. For comparison, we analyzed a sample of relaxed clusters of comparable mass and redshift. Considering the magnitude limit of SDSS photometry, the typical redshift of the samples, $z \sim 0.25$, limits our study to the bright part of the LF. We plan to extend this study to fainter magnitudes in the near future.

Unless otherwise stated, we give errors at the $68 \%$ confidence level (hereafter c.l.).

Throughout this paper, we use $H_{0}=70 \mathrm{~km} \mathrm{~s}^{-1} \mathrm{Mpc}^{-1}$ in a flat cosmology with $\Omega_{0}=0.3$ and $\Omega_{\Lambda}=0.7$. We define $h_{70}=$ $H_{0} /\left(70 \mathrm{~km} \mathrm{~s}^{-1} \mathrm{Mpc}^{-1}\right)$.

\section{Cluster sample}

Among the DARC clusters of which we have already analyzed the internal dynamics using a large number of member galaxies, five clusters (with 62-113 spectroscopic member galaxies) have also available photometry in the SDSS (Data Release 7): Abell 697, Abell 773, Abell 959, Abell 1240, and Abell 2219, hereafter A697, A773, A959, A1240, A2219 (see Table 1 for the respective reference sources). Their redshifts span the range $z=0.19-0.29$ and they are characterized by high line-of-sight galaxy (LOS) velocity dispersion $\sigma_{\mathrm{v}}, \mathrm{X}$-ray temperature $T_{\mathrm{X}}$, and mass $M$ (see Table 1). Evidence for their unrelaxed dynamical state is reported in our previous studies. In particular, they span different angles of view for the merging axis. For instance, the cluster merger occurred mainly in the plane of the sky in A1240 and mainly along the LOS in A773.

For comparison, we select a few well-known examples of very relaxed clusters from the literature spanning a similar $z$

\footnotetext{
1 Other information is given on the web site http://adlibitum. oat.ts.astro.it/girardi/darc
} 
range $(0.2 \lesssim z \lesssim 0.3)$, characterized by high $T_{\mathrm{X}}$, and sampled by SDSS. From the relaxed clusters listed by Allen et al. (2004) we selected $^{2}$ Abell 383, Abell 963, and Abell 1835 (hereafter A383, A963, A1835). These clusters are all classified as cool-core clusters according to Baldi et al. (2007). This agrees with the observations that suggest that cool cores are destroyed by cluster mergers and cool-core clusters are in the final phase of cluster relaxation (see e.g., Allen et al. 2001; Buote 2002; Sanderson et al. 2006). Among the cool-core clusters listed by Baldi et al. (2007) we considered another two cool-core clusters to complete our comparison sample: Abell 2261 (hereafter A2261) and ZwCl 1021.0+0426 (hereafter and better known as ZwC13146).

To avoid the inherent bias that has plagued numerous studies (see the section above), the cluster LFs will be compared based on scaling relative to the dynamical radius, $R_{200}$. As an estimate of $R_{200}$, we used $R_{\text {vir }}$ as defined by Girardi \& Mezzetti (2001), Eq. (1) with the scaling of $H(z)$,

$R_{200}=0.17 \times \sigma_{\mathrm{v}} /\left(\mathrm{km} \mathrm{s}^{-1}\right) / H(z) h_{70}^{-1} \mathrm{Mpc}$,

where $\sigma_{\mathrm{v}}$ is the LOS velocity dispersion. The same result was obtained by Carlberg et al. (1997, see their Eq. (8) for $R_{200}$ ) using a different, more theoretical, approach. Biviano et al. (2006) obtained a $10 \%$ lower estimate of $R_{200}, R_{\mathrm{v}}=0.15 \times$ $\sigma_{\mathrm{v}} /\left(\mathrm{km} \mathrm{s}^{-1}\right) / H(z) h_{70}^{-1} \mathrm{Mpc}$ on the base of $N$-body simulations. We also considered internal and external regions, i.e. $R<0.5 R_{200}$ and $0.5 R_{200}<R<R_{200}$, respectively. The centers for relaxed clusters are taken from X-ray studies, while those for DARC clusters are those used in our previous papers. Owing to the unrelaxed state of DARC clusters, the choice of the cluster center is not obvious since gas and galaxy spatial distributions are generally different. For A697, A773 and A2219 clusters we used the position of the brightest dominant galaxy, very close to the X-ray centroid (or X-ray main peak), while for A959 and A1240 we used the X-ray centroid. However, in these two cases, the X-ray centroid is located in between the two brightest dominant galaxies. A radius of $0.5 R_{200}$ is sufficient to contain the cores of galaxy subclusters, the most critical case being the bimodal cluster A1240, which is strongly elongated in the plane of the sky.

One can use the measured global value of the velocity dispersion, $\sigma_{\mathrm{v}, \text { tot }}$, to estimate the mass, $M_{200}$, in a relaxed cluster within $R_{200}$. The estimation of mass is based on the virial theorem and we specifically follow the prescriptions of Girardi et al. (1998; see also Girardi \& Mezzetti 2001). We refer the reader to the original papers for the details, but, in practice, one can use

$M_{200}=A \times\left[\sigma_{\mathrm{v}} /\left(10^{3} \mathrm{~km} \mathrm{~s}\right)\right]^{3} \times h_{70}^{-1} 10^{15} M_{\odot}$,

where $A \sim 1.4$ (here median $A=1.416$ ). Thus both our estimates of $R_{200}$ and $M_{200}$ depend on the estimate of the observable value of $\sigma_{v}$. However, they are not mutually consistent according to the definition $M_{200}=200 \times \rho_{\text {crit }} \times 4 / 3 \times \pi \times R_{200}^{3}$, where $\rho_{\text {crit }}$ is the critical density of the Universe at the cluster redshift. Rather, according to the definition, they correspond to $R_{194}$ and $M_{194}$.

We estimate $\sigma_{\mathrm{v}}$ from the observed $T_{\mathrm{X}}$ for the relaxed clusters assuming the equipartition of energy density between ICM and galaxies, i.e. $\beta_{\text {spec }}=1$, where $\beta_{\text {spec }}=\sigma_{\mathrm{V}}^{2} /\left(k T / \mu m_{\mathrm{p}}\right)$ with $\mu=$ 0.58 the mean molecular weight and $m_{\mathrm{p}}$ the proton mass. This assumption is particularly appropriate for massive clusters (e.g., Girardi et al. 1996, 1998). $R_{200}$ and $M_{200}$ are thus recovered with the above equations.

\footnotetext{
${ }^{2}$ Note that we excluded Abell 611 because of its unrelaxed appearance in the SDSS image.
}

For each relaxed cluster, Table 2 lists the main properties: the cluster center (Col. 2); the cluster redshift, $z$ (Col. 3); the X-ray temperature, $T_{\mathrm{X}}(\mathrm{Col} .4)$; the estimated value of $\sigma_{\mathrm{v}}$ from $T_{\mathrm{X}}$ (Col. 5); the values of $R_{200}$ and $M_{200}$ (Cols. 6 and 7); useful references (Col. 8).

We adopt a more complex procedure for the DARC clusters. During a cluster merger, the global value of velocity dispersion, $\sigma_{\mathrm{v}, \text { tot }}$, might strongly vary and be only a very poor indicator of the real cluster potential (e.g., Pinkney et al. 1996), and the same problem could afflict $T_{\mathrm{X}}$ (e.g., Ricker \& Sarazin 2001; Mastropietro \& Burkert 2008). As discussed in our papers for the five DARC clusters, we have detected the main subclusters and obtained an estimate of their individual velocity dispersion, $\sigma_{\mathrm{v}, \text { subs }}$. Therefore, an alternative, likely more reliable value of the mass $M_{200}$ of the cluster can then be obtained by adding the masses of all subclusters. Then, inverting the above scaling relation Eq. (2), we obtained a value of the velocity dispersion, $\sigma_{\mathrm{v}}$, which should be a better indicator of the potential. The use of Eq. (1) leads to the estimate of $R_{200}$.

For each DARC cluster, Table 1 lists the main properties: the cluster center (Col. 2); the cluster redshift, $z$ (Col. 3); the X-ray temperature, $T_{\mathrm{X}}$ (Col. 4); the global observed value of velocity dispersion, $\sigma_{\mathrm{v}, \mathrm{obs}}(\mathrm{Col} .5)$; the (rounded) values of velocity dispersions for subclusters $\sigma_{\mathrm{v} \text {,subs }}(\mathrm{Col} .6)$; the estimated value of mass from the addition of subclusters, $M_{200}$ (Col. 7); the corresponding values of $\sigma_{\mathrm{v}}$ and $R_{200}$ (Cols. 8 and 9). The values of the cluster center, $z, T_{\mathrm{X}}, \sigma_{\mathrm{v}, \text { tot }}$, and $\sigma_{\mathrm{v} \text {,subs }}$ can be obtained from the listed references. In particular, the notes list the source for the values of $\sigma_{\mathrm{v}, \text { subs }}$, here rounded. Other values are homogeneously computed in this study.

\section{Magnitude data and background contamination}

The magnitude data $\left(r^{\prime}, i^{\prime}\right)$ are taken from SDSS (Data Release 7). We retrieved model mag values. For each cluster, we created a galaxy "cluster galaxy sample" by selecting the galaxies in an area centered on the cluster center listed in Tables 2 and 1 and within the respective radius $R_{200}$. We verified that the whole cluster area is covered by SDSS data by visual inspection.

For each cluster, we also considered a galaxy sample selected in one square degree field at 1.5 degree toward the west of each cluster and obtained ten individual field samples. Together these samples, for a total area of ten square degrees, forms the "field galaxy sample" and are used to estimate the field galaxy contamination. Figure 1 shows the field counts for $r^{\prime}$ and $i^{\prime}$ magnitudes, and the logarithmic function fit. This fit represents the countmagnitude relation expected for a homogeneous galaxy distribution in a universe with Euclidean geometry. We obtain the relations $\log \left(N_{r}\right)=0.455( \pm 0.043) r^{\prime}-(6.17 \pm 0.59) 0.5 \mathrm{mag}^{-1} \mathrm{deg}^{-2}$ for $15<r^{\prime}<22$ and $\log \left(N_{i}\right)=0.451( \pm 0.046) i^{\prime}-(5.70 \pm$ 0.58) $0.5 \mathrm{mag}^{-1} \mathrm{deg}^{-2}$ for $15<i^{\prime}<21.5$. We used these fits with two purposes: i) to estimate the completeness of our sample; ii) to subtract field galaxy contamination in each cluster.

The completeness magnitude can be derived through comparison with the logarithmic fits. We estimate the photometric sample be complete if the magnitude counts are lower than 5\% of the fit. Therefore, we consider the $r^{\prime}$ - and $i^{\prime}$-band photometry complete down to $r^{\prime}=22.4$ and $i^{\prime}=21.4$, respectively. We find that these values agree very well with those reported by the Sloan Digital Sky Survey DR7 (see http://www.sdss.org/ $\operatorname{dr} 7 /$ ).

Two different approaches to the statistical subtraction of the galaxy foreground/background are discussed in the literature: several studies have examined the effect on the derived cluster 
Table 1. Unrelaxed clusters (DARC sample).

\begin{tabular}{|c|c|c|c|c|c|c|c|c|}
\hline Name & $\alpha, \delta(\mathrm{J} 2000)$ & $z$ & $\begin{array}{c}T_{\mathrm{X}} \\
\mathrm{keV}\end{array}$ & $\begin{array}{c}\sigma_{\mathrm{v}, \mathrm{tot}} \\
\mathrm{km} \mathrm{s}^{-1}\end{array}$ & $\begin{array}{c}\sigma_{\mathrm{v}, \text { subs }} \\
\left(\mathrm{km} \mathrm{s}^{-1}\right)\end{array}$ & $\begin{array}{c}M_{200} \\
10^{15} M_{\odot}\end{array}$ & $\begin{array}{c}\sigma_{\mathrm{v}} \\
\left(\mathrm{km} \mathrm{s}^{-1}\right)\end{array}$ & $\begin{array}{l}R_{200} \\
\mathrm{Mpc}\end{array}$ \\
\hline $\mathrm{A} 697^{a}$ & $084257.55,+362159.9$ & 0.2815 & 10.2 & $1334_{-95}^{+114}$ & $200,600,400$ & 0.40 & 660 & 1.39 \\
\hline $\mathrm{A} 773^{b}$ & $091753.26,+514336.5$ & 0.2197 & 7.8 & $1394_{-68}^{+84}$ & 950,500 & 1.39 & 994 & 2.16 \\
\hline A959 ${ }^{c}$ & $101735.04,+593327.7$ & 0.2883 & 7.0 & $1170_{-73}^{+83}$ & 600,700 & 0.76 & 824 & 1.73 \\
\hline $\mathrm{A} 1240^{d}$ & $112337.60,+430551.0$ & 0.1948 & 6.0 & $870_{-79}^{+91}$ & 700,1000 & 1.93 & 1103 & 2.43 \\
\hline $\mathrm{A} 2219^{e}$ & $164019.87,+464241.3$ & 0.2254 & 10.3 & $1438_{-86}^{+109}$ & 1000 & 1.41 & 1000 & 2.17 \\
\hline
\end{tabular}

Notes. ${ }^{(a)}$ Ref.: Girardi et al. (2006); $\sigma_{\mathrm{v}, \text { subs }}$ from KMM4g1-2-4 in Table 4. ${ }^{(b)}$ Ref.: Barrena et al. (2007); $\sigma_{\mathrm{v}, \text { subs }}$ from the main and secondary subclusters in Sect. 5. ${ }^{\left({ }^{c}\right)}$ Ref.: Boschin et al. (2008); $\sigma_{\mathrm{v}, \text { subs }}$ from the averages of KMM1 and V1, and of KMM2 and V2 in Table 2. Here we do not consider the subclump V3(=KMM3=DS-NE) also detected in X-ray, which is likely in a premerging phase and not responsible for the ongoing merger. ${ }^{(d)}$ Ref.: Barrena et al. (2009); $\sigma_{\mathrm{v}, \text { subs }}$ from A1240N and A1240S in Table 2. ${ }^{(e)}$ Ref.: Boschin et al. (2004); $\sigma_{\mathrm{v}, \text { subs }}$ from NW in Table 2. The separation of suclumps is not obvious. Here, since the primary subclump lies to the SE of the cluster center (see also the more recent paper Million \& Allen 2009), we consider the value of $\sigma_{\mathrm{v}}$ of NW sector, where the main cluster is likely free of the subclump.

Table 2. Relaxed cluster (comparison sample).

\begin{tabular}{lccccccc}
\hline \hline Name & $\alpha, \delta(\mathrm{J} 2000)$ & $z$ & $\begin{array}{c}T_{\mathrm{X}} \\
\mathrm{keV}\end{array}$ & $\begin{array}{c}\sigma_{\mathrm{v}} \\
\left(\mathrm{km} \mathrm{s}^{-1}\right)\end{array}$ & $\begin{array}{c}R_{200} \\
\mathrm{Mpc}\end{array}$ & $\begin{array}{c}M_{200} \\
10^{15} M_{\odot}\end{array}$ & Refs. $^{a}$ \\
\hline A383 & $024803.50,-033145.0$ & 0.188 & 3.9 & 802 & 1.78 & 0.74 & Allen et al. (2004), Maughan et al. (2008) \\
A963 & $101701.20,+390144.4$ & 0.206 & 6.0 & 995 & 2.18 & 1.41 & Allen et al. (2004), Baldi et al. (2007) \\
A1835 & $140102.40,+025255.2$ & 0.252 & 8.1 & 1156 & 2.47 & 2.15 & Allen et al. (2004), Baldi et al. (2007) \\
A2261 & $172227.60,+320757.2$ & 0.224 & 7.2 & 1090 & 2.36 & 1.83 & Baldi et al. (2007), Maughan et al. (2008) \\
ZwCl3146 & $102339.60,+041124.0$ & 0.291 & 8.6 & 1191 & 2.49 & 2.31 & Baldi et al. (2007), Baldi et al. (2007) \\
\hline
\end{tabular}

Notes. ${ }^{(a)}$ References for $z$ and $T_{\mathrm{X}}$ are listed in the first and second position, respectively. Cluster centers are taken from Ebeling et al. (1996) or Ebeling et al. (1998).

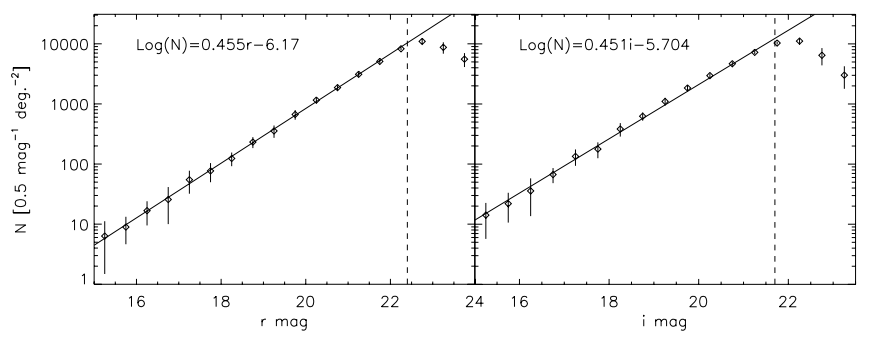

Fig. 1. Values of $r^{\prime}$ - and $i^{\prime}$-band counts for the 10 square degrees considered as the field galaxy sample. The solid line indicates the logarithmic function fit to the histograms. The dashed line indicates the completeness magnitudes.

LF using a global galaxy field correction versus one measured locally for each cluster. For instance, Popesso et al. (2004) have shown for a study of about 100 clusters based on SDSS data that there is no significant difference in the measured cluster LF parameters using either a global or local field subtraction technique. Here we consider the global field correction, subtracting the field counts derived from the logarithmic fits to the cluster counts. Note that the field counts are treated in a different and appropriate way for each cluster before the subtraction.

The magnitudes of each cluster were corrected for the galactic absorption using the Schlegel, Finkbeiner and Davis Galactic reddening maps (Schlegel et al. 1998), derived from IRAS and COBE/DIRBE data.

The possible contamination of distant clusters projected onto the field-of-view of the target cluster can prove to be problematic in the derivation of the LF, in particular at the faint-end. One can
Table 3. Color-magnitude relations (CMR) $r^{\prime}-i^{\prime}$ vs. $r^{\prime}$ linear fits $(a \dot{z}+b)$ : red sequences.

\begin{tabular}{lcc}
\hline \hline Name & $(a, \delta a)$ & $(b, \delta b)$ \\
\hline A697 & $-0.0107,0.014$ & $0.744,0.041$ \\
A773 & $-0.0213,0.007$ & $0.861,0.037$ \\
A959 & $-0.0310,0.006$ & $1.126,0.037$ \\
A1240 & $-0.0110,0.020$ & $0.666,0.037$ \\
A2219 & $-0.0086,0.007$ & $0.668,0.062$ \\
\hline A383 & $-0.0160,0.001$ & $0.763,0.047$ \\
A963 & $-0.0422,0.007$ & $1.216,0.042$ \\
A1835 & $-0.0004,0.011$ & $0.501,0.031$ \\
A2261 & $-0.0091,0.015$ & $0.653,0.048$ \\
ZwCl3146 & $-0.0220,0.003$ & $1.020,0.050$ \\
\hline
\end{tabular}

use that the spectroscopic studies show that there are essentially no cluster galaxies significantly redward of the red sequence (e.g. Rines \& Geller 2008). We minimized the contamination of background clusters using the $r^{\prime}-i^{\prime}$ vs. $r^{\prime}$ colour-magnitude relation, which has proved to be the most useful for target clusters at $0.2<z<0.4$ (Lu et al. 2009, their Fig. 6 and Sect. 3.4.1). For each cluster, we fitted the red sequence (RS) $r^{\prime}-i^{\prime}$ vs. $r^{\prime}$ for galaxies. We rejected galaxies that are 0.15 mag redward of the RS (i.e. $\gtrsim 3$ times the average dispersion of the cluster RS). Figure 2 shows an example for the RS of A383. After this background correction, the clusters appear more contrasted with respect to the surrounding field (see Fig. 3 for A383).

Table 3 lists, for each cluster, the slope $a$ and the intercept $b$ and standard deviations of the RS $r^{\prime}-i^{\prime}$ vs. $r^{\prime}$ obtained with a linear fit within $0.5 h_{70}^{-1} \mathrm{Mpc}$ from the center and for galaxies with $r^{\prime}<20.2$ (Cols. 2 and 3). 


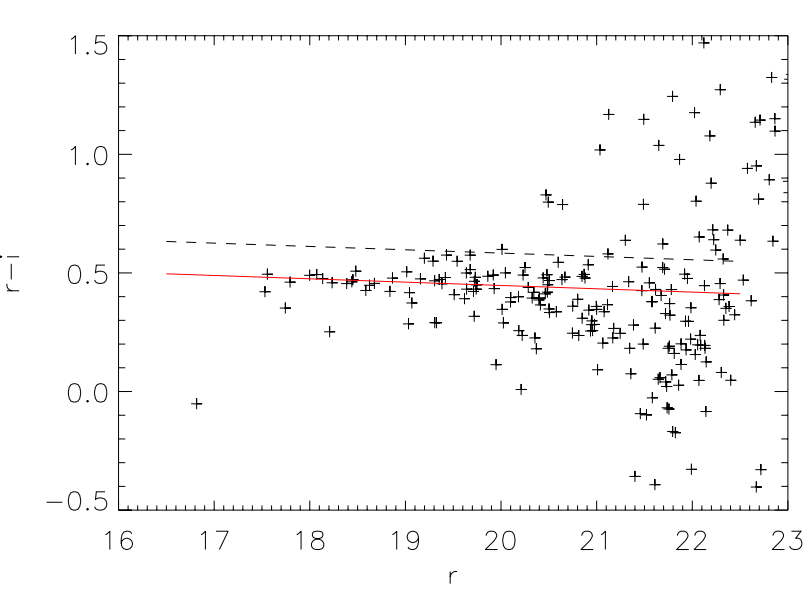

Fig. 2. Color-magnitude diagram $r^{\prime}-i^{\prime}$ vs. $r^{\prime}$ considering galaxies within $0.5 h_{70}^{-1} \mathrm{Mpc}$ from the center of Abell 383. The RS (red solid line) is fitted using galaxies with $r^{\prime}<20.2$. Galaxies with $r^{\prime}-i^{\prime}>$ $\mathrm{RS}+0.15$ (on the dashed line) are considered background galaxies and were rejected from the analysis.

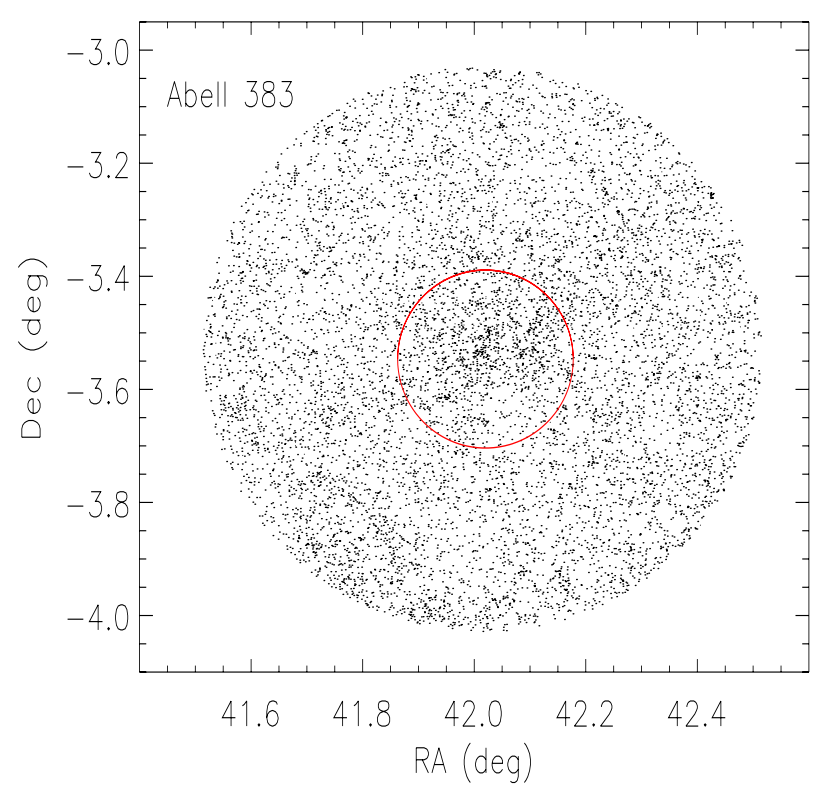

Fig. 3. Galaxies with $r^{\prime}-i^{\prime}<\mathrm{RS}+0.15$ in the region of A383. The red circle indicates $R_{200}$ for this cluster.

Figure 4 shows the RS parameters vs. cluster redshift. There is no correlation with $z$ in the observed range for the RS slope and we estimate a mean value of $\langle a\rangle=-0.017 \pm 0.008$. For the $r^{\prime}-i^{\prime}$ colors at $r^{\prime}=17$ computed from the RS fits, there is no difference in the behavior of DARC and relaxed clusters: we only observe a marginally higher value for the most distant cluster A697, A959 and ZwCl3146, which is consistent with the $k$-correction effect on early-type galaxies in the central core of clusters (see Fukugita et al. 1995, Tables 3, 6, 7 and 8). Assuming a linear variation of the color with the redshift, we obtain the relation $\left(r^{\prime}-i^{\prime}\right)_{r^{\prime}=17}=1.24 * z+0.23$, with a global dispersion in color of \pm 0.02 .

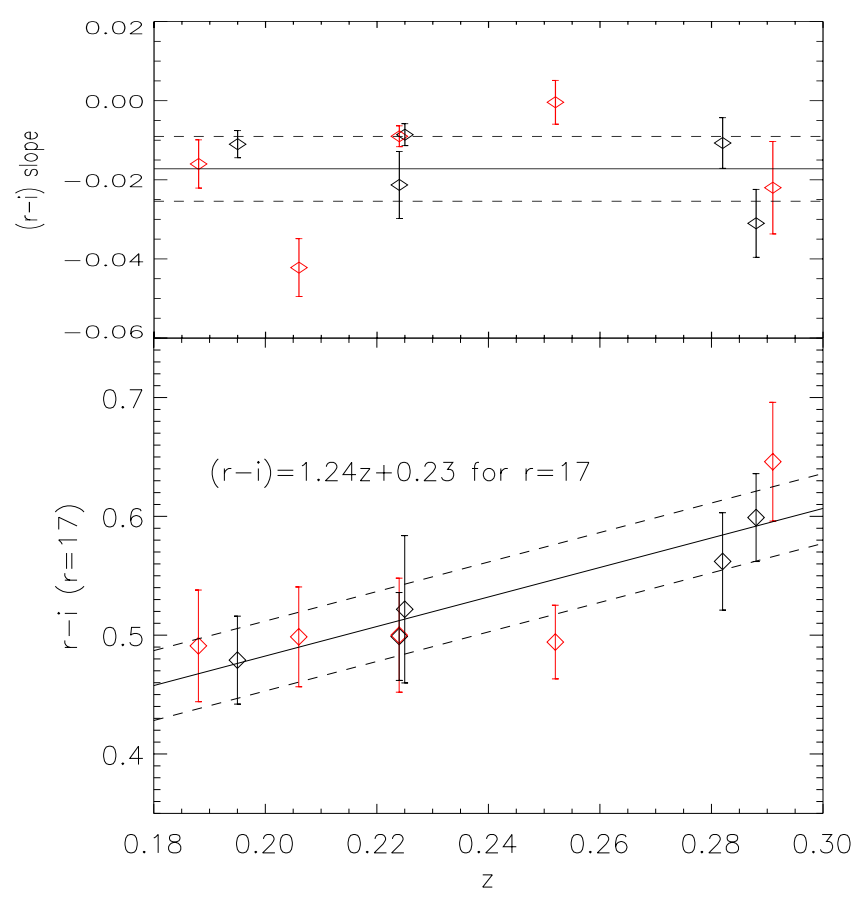

Fig. 4. RS parameters vs. redshift: the $\left(r^{\prime}-i^{\prime}\right)$ vs. $r^{\prime}$ slope (upper panel) and $\left(r^{\prime}-i^{\prime}\right)$ color at $r^{\prime}=17$ (lower panel). Black symbols indicate DARC clusters and red symbols indicate relaxed clusters. The solid line in the upper panel shows the mean value for all ten clusters. The solid line in the lower panel shows the linear fit. The dashed lines show onesigma uncertainties of the linear fit.

For each cluster, we transformed the galaxy apparent into absolute magnitudes by applying the relation

$M_{r}=r^{\prime}-2.5-5 \log \left(D_{L} / 1 \mathrm{Mpc}\right)-K(z)+E(z)$,

where $r^{\prime}$ is the apparent magnitude (already corrected for the Galactic absorption, see above), $D_{L}$ is the luminosity distance; $K(z)$ is the K-correction and $E(z)$ is the evolutionary correction. $K(z)$ and $E(z)$ corrections were applied using a single parametrization based on early-type galaxies, which are dominant in the cluster galaxy population. For $K(z)$, we used the correction obtained from Table 2, Col. 3 in Roche et al. (2009). For the evolutionary correction, we used $E(z)=0.86 z$ (Roche et al. 2009). Given that the completeness of the photometric sample is $r^{\prime}=22.4$ (Fig. 1) and the most distant cluster of our catalogs is at $z=0.29$, we expect that the LFs we obtain in the present study are all reliable for an absolute magnitude $M_{r}<-18.6$.

Before the field subtraction in the construction of the LF (see below), the field galaxies of each cluster were treated in the same way as the respective cluster galaxies, i.e. we applied the same correction for the Galactic absorption, the same rejection of very red galaxies and the same transformation in absolute magnitudes.

\section{Galaxy populations in DARC and relaxed clusters}

\subsection{Individual luminosity functions}

Figure 5 shows the individual LFs for DARC and relaxed clusters within $R_{200}$, where the galaxy counts are computed as $N_{i}=N_{\text {net }, i} / N_{\text {net, }<-20} . N_{\text {net }, i}$ are the net counts, i.e. $N_{\text {net, } i}=$ $N_{\text {cluster,i }}-N_{\text {field, } i}$ where the field counts are conveniently normalized to the cluster area. $N_{\text {net, }<-20}$ is the number of net counts for 


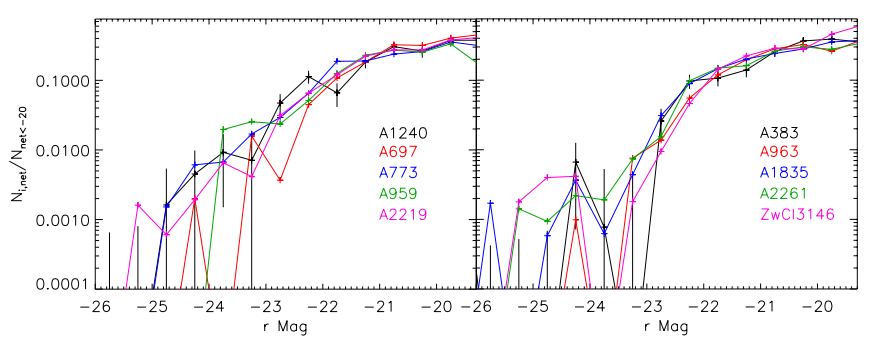

Fig. 5. LFs for DARC clusters (right panel) and relaxed clusters (left panel) as computed within $R_{200}$. Error bars are only plotted in the A1240 and A383 LFs for the sake of clarity.

Table 4. $M_{r}^{\star}$ and $\alpha$ values obtained from Schechter fits for the LF.

\begin{tabular}{lcccc}
\hline \hline & \multicolumn{2}{c}{$R<R_{200}$} & \multicolumn{2}{c}{$R<0.5 R_{200}$} \\
Name & $M_{r}^{\star}, \Delta M_{r}^{\star}$ & $\alpha, \Delta \alpha$ & $M_{r}^{\star}, \Delta M_{r}^{\star}$ & $\alpha, \Delta \alpha$ \\
\hline A697 & $-21.19,0.30$ & $-0.96,0.20$ & $-20.95,0.16$ & $-0.70,0.21$ \\
A773 & $-21.84,0.26$ & $-1.01,0.13$ & $-21.43,0.24$ & $-0.61,0.18$ \\
A959 & $-21.60,0.62$ & $-0.96,0.39$ & $-22.00,0.94$ & $-0.96,0.46$ \\
A1240 & $-22.31,0.36$ & $-1.28,0.12$ & $-21.61,0.37$ & $-0.95,0.20$ \\
A2219 & $-21.74,0.28$ & $-1.09,0.15$ & $-21.79,0.08$ & $-0.97,0.08$ \\
\hline A383 & $-21.89,0.25$ & $-1.30,0.17$ & $-21.92,0.27$ & $-1.26,0.21$ \\
A963 & $-21.31,0.28$ & $-0.83,0.19$ & $-20.93,0.22$ & $-0.27,0.25$ \\
A1835 & $-21.78,0.21$ & $-1.02,0.15$ & $-22.06,0.33$ & $-1.13,0.18$ \\
A2261 & $-21.56,0.22$ & $-0.88,0.16$ & $-21.62,0.25$ & $-1.01,0.19$ \\
ZwC13146 & $-21.36,0.26$ & $-0.97,0.24$ & $-20.89,0.23$ & $-0.51,0.29$ \\
\hline
\end{tabular}

$M_{r}<-20$ that represents the "richness", which is used here to normalize. The error bars in Fig. 5 are the richness-normalized $\sigma_{i}=\left(N_{i}+N_{b, i}+\mathrm{rms}_{f}^{2}\right)^{1 / 2}$, where the first two terms take into account the Poissonian errors of the cluster and background field samples, and the third term measures the field-to-field variation per bin in the background field counts (i.e., the cosmic variance obtained using the ten individual field samples). In order to see possible variations with the radius, we also computed the LFs within $0.5 R_{200}$ and $R_{200}$.

Each LF was fitted to a Schechter function (see Schechter 1976) using the "minuit" procedure (CERN Libraries) to minimize the three values, $\phi$ (richness), $\alpha$ (slope) and $M^{*}$ of the Schechter profile. We do not discuss the $\phi$ parameter since here the counts are already normalized by the "richness" $N_{\text {net },<-20}$ for each cluster. Table 4 shows the $M^{*}$ and $\alpha$ values obtained for each cluster, fitting Schechter functions within $R_{200}$ and $0.5 R_{200}$.

Figure 6 shows $\alpha$ vs. $M^{*}$ values estimated for each cluster within $R_{200}$ : evidently there is no difference between DARC and relaxed clusters. We obtain the same result for LFs within $0.5 R_{200}$ and when comparing $R_{200}$ and $0.5 R_{200}$ results.

For the DARC sample we obtain a mean value of $M_{\mathrm{DARC}}^{*}=$ $-21.73 \pm 0.16$ and $-21.57 \pm 0.16$, considering galaxies within $R_{200}$ and $0.5 R_{200}$, respectively. On the other hand, for the relaxed clusters, we derive $M_{\text {rel }}^{*}=-21.58 \pm 0.11$ and $-21.48 \pm 0.12$. For the slope parameter, we estimate $\alpha_{\text {DARC }}=-1.06 \pm 0.09$ and $-0.84 \pm 0.10$ within $R_{200}$ and $0.5 R_{200}$, respectively. For the relaxed clusters, we obtain $\alpha_{\text {rel }}=-1.00 \pm 0.12$ and $-0.84 \pm 0.10$. Owing to the correlated nature of $\alpha$ and $M^{\star}$ parameters in the fitting procedure, which likely leads to the apparent correlation in Fig. 6, we prefered to compare individual clusters by fixing one parameter and repeating the fit. When fixing $\alpha=-1.00$ for the slope parameter for all individual clusters, we found no longer any difference (see Table 5). In particular, when fixing the slope, we obtain $M_{\text {DARC }}^{*}=-21.64 \pm 0.06$ and $M_{\text {rel }}^{*}=-21.61 \pm 0.05$
Table 5. $M_{r}^{\star}$ values obtained from the Schechter fit when fixing $\alpha=$ -1.00 for the LF.

\begin{tabular}{lcc}
\hline \hline & $R<R_{200}$ & $R<0.5 R_{200}$ \\
Name & $M_{r}^{\star}, \Delta M_{r}^{\star}$ & $M_{r}^{\star}, \Delta M_{r}^{\star}$ \\
\hline A697 & $-21.30,0.12$ & $-21.19,0.79$ \\
A773 & $-21.85,0.11$ & $-22.15,0.12$ \\
A959 & $-21.83,0.16$ & $-22.25,0.19$ \\
A1240 & $-21.65,0.17$ & $-21.69,0.18$ \\
A2219 & $-21.57,0.12$ & $-21.81,0.31$ \\
\hline A383 & $-21.51,0.14$ & $-21.65,0.21$ \\
A963 & $-21.54,0.12$ & $-21.70,0.13$ \\
A1835 & $-21.75,0.09$ & $-21.95,0.14$ \\
A2261 & $-21.72,0.09$ & $-21.59,0.11$ \\
ZwC13146 & $-21.53,0.13$ & $-21.33,0.08$ \\
\hline
\end{tabular}

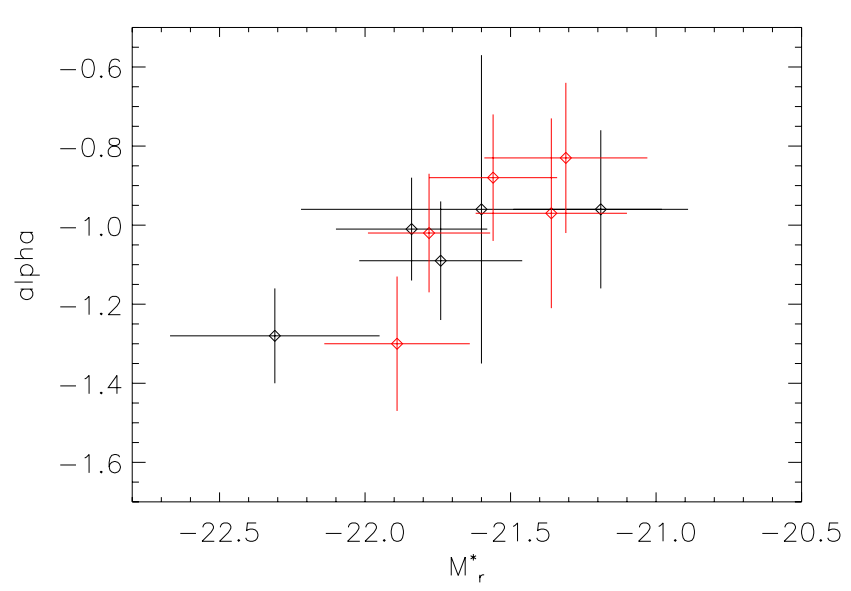

Fig. 6. $\alpha$ vs. $M_{r}^{\star}$ derived from the Schechter LF fit. Black symbols indicate DARC clusters and red symbols indicate relaxed clusters.

within $R<R_{200}$ and $M_{\mathrm{DARC}}^{*}=-21.82 \pm 0.14$ and $M_{\mathrm{rel}}^{*}=$ $-21.64 \pm 0.06$ within $R<0.5 R_{200}$. From these results, we can conclude that the global LF profile is similar for DARC and relaxed clusters, independently of the sampling radius.

The mean LF will be studied in the following subsection, and we will detail possible differences considering blue and red galaxy populations in the inner and external regions.

\subsection{The composite luminosity functions}

For both DARC and relaxed clusters we separately constructed the composite LFs by combining them into a single "ensemble" LF, to improve the rather poor number statistics for each individual LF. The composite LF was built by averaging, in absolute magnitude bins, the richness-normalized net counts as obtained above for each individual LF. We assumed the standard errors on the average.

Figure 7 shows the composite LFs for DARC and relaxed clusters within $R_{200}$ and $0.5 R_{200}$. Table 6 summarizes the $M^{*}$ and $\alpha$ parameters obtained for the composite LFs. In agreement with the above section, there is no evidence of difference between DARC and relaxed samples and there is no dependence on the sampling radius. 


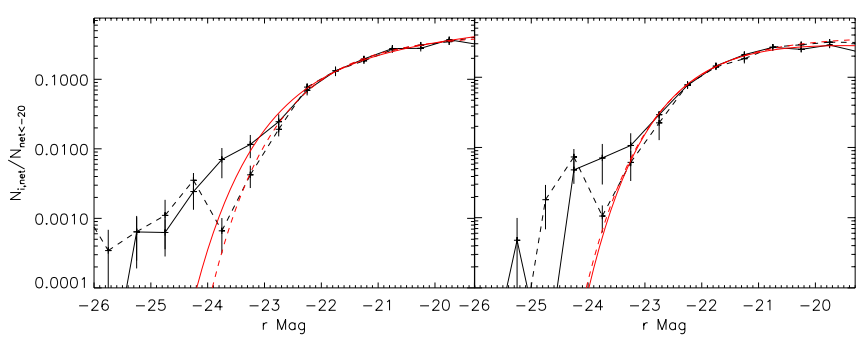

Fig. 7. Composite LFs within $R_{200}$ (left panel) and $0.5 R_{200}$ (right panel). Solid lines show the counts for DARC clusters and dashed lines show the counts for the relaxed clusters. The red lines indicate the corresponding Schechter fits.

Table 6. $M^{*}$ and $\alpha$ for the composite LFs.

\begin{tabular}{lcccc}
\hline \hline \multicolumn{5}{c}{$R<R_{200}$} \\
Whole galaxy population \\
Sample & $M^{*}, \delta M^{*}$ & $\alpha, \Delta \alpha$ & $M^{*}, \delta M^{*}$ & $\alpha, \Delta \alpha$ \\
\hline DARC & $-22.00,0.41$ & $-1.20,0.17$ & $-21.64,0.35$ & $-0.86,0.27$ \\
Relaxed & $-21.63,0.24$ & $-1.03,0.20$ & $-21.74,0.29$ & $-0.99,0.21$ \\
\multicolumn{5}{c}{ Red galaxies } \\
\hline DARC & $-21.62,0.25$ & $-0.76,0.22$ & $-21.23,0.28$ & $-0.47,0.27$ \\
Relaxed & $-21.51,0.28$ & $-0.76,0.23$ & $-21.50,0.36$ & $-0.70,0.30$ \\
& & Blue galaxies & \\
\hline DARC & $-22.55,0.27$ & $-2.03,0.10$ & $-22.98,0.37$ & $-1.79,0.24$ \\
Relaxed & $-21.66,0.52$ & $-1.44,0.46$ & $-23.63,0.53$ & $-2.07,0.23$ \\
\hline
\end{tabular}

Figure 7 shows the Schechter functions fit the counts for galaxies with $M_{r^{\prime}}>-22.5$ reasonably well. We obtain ${ }^{3} \chi^{2}=$ 0.45 for DARC clusters and 0.35 for relaxed clusters (both within $R_{200}$ ) and $\chi^{2}=0.33$ for DARC clusters and 0.27 for relaxed clusters (both within $0.5 R_{200}$ ). On the other hand, the bright-end $\left(M_{r^{\prime}}<-22.5\right)$ is not satisfactorily represented by Schechter functions. In any case, we obtain $\chi^{2}$ values higher than 1.4. Therefore, the contribution of these bright galaxies (hereafter BGs) have to be analyzed separately (see Sect. 5).

\subsection{Blue and red LFS}

We also estimated the LF for red and blue galaxies within $R_{200}$ and $0.5 R_{200}$. We selected red and blue galaxies from the RS (see above). We assumed that red galaxies are those objects within the RS, that have RS-0.15 $<r^{\prime}-i^{\prime}<\mathrm{RS}+0.15$, while blue galaxies were selected as $r^{\prime}-i^{\prime}<\mathrm{RS}-0.15$. Results are presented in Fig. 8 and summarized in Table 6.

When considering the whole galaxy population, no differences are found between DARC and relaxed clusters within $R_{200}$ or inside the core of the clusters, within $0.5 R_{200}$. The differences in $M^{\star}$ and $\alpha$ are not significant and within $3 \sigma$ errors (see Fig. 9). The same is also true for the red galaxy population. We only note a marginal difference in the LF parameters of blue galaxies, in particular in the core of the clusters, inside $0.5 R_{200}$.

To check the significance of these differences, we fitted the Schechter profiles to the composite LFs by fixing the slope parameter. Table 7 lists the results. Again, we confirm that there are no differences between DARC and relaxed LFs. As above, we only find small differences for the blue galaxy population

\footnotetext{
3 The $\chi^{2}$ statistic has been calculated considering three degrees of freedom: the normalization coefficient, $M^{\star}$ and the slope.
}
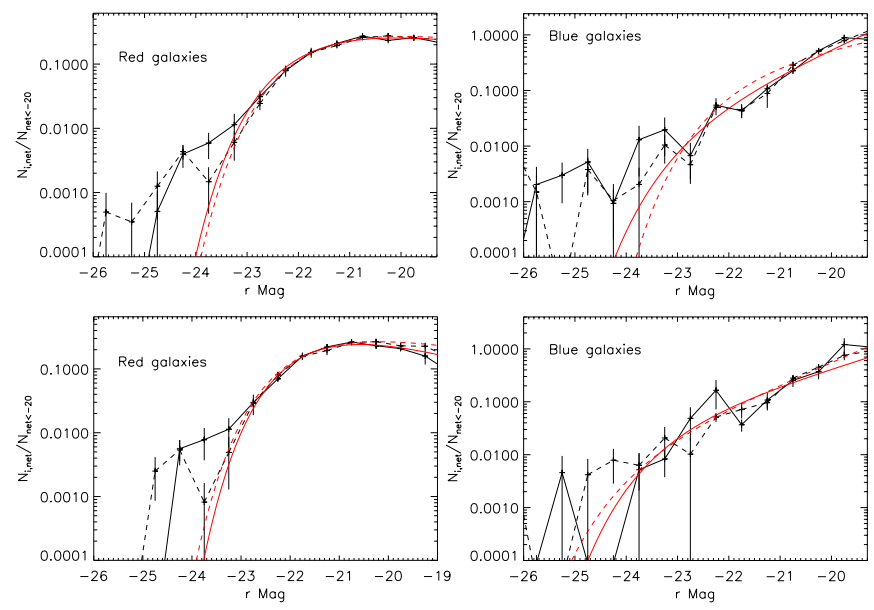

Fig. 8. Upper panels: LFs and Schechter fits for red and blue galaxies computed within $R_{200}$. Lower panels: the same within $0.5 R_{200}$. Solid lines indicate DARC clusters and dashed lines indicate relaxed clusters.
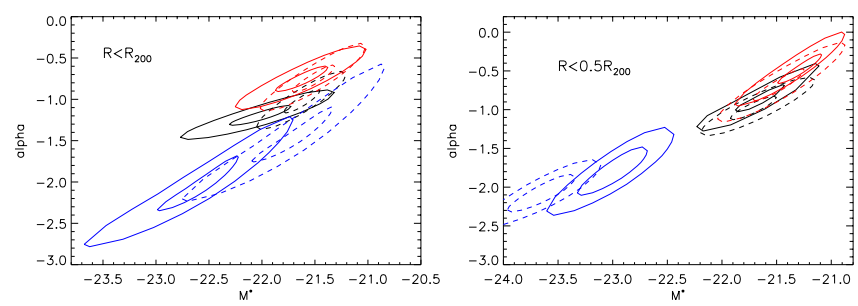

Fig. 9. Error contours at the 1 and $3 \sigma$ c.l. for the best-fitting LF parameters considering the galaxy population within $R<R_{200}$ (left panel) and $R<0.5 R_{200}$ (right panel). Black, red and blue lines show the composite LF for the whole galaxy sample, red and blue galaxies, respectively. Solid lines indicate DARC clusters and dashed lines indicate relaxed clusters.

Table 7. $M^{*}$ for the composite LFs (at fixed $\alpha$ ).

\begin{tabular}{|c|c|c|}
\hline & $R<R_{200}$ & $R<0.5 R_{200}$ \\
\hline $\begin{array}{l}\text { Whole g } \\
\text { Sample }\end{array}$ & $\begin{array}{c}\text { laxy population } \\
M^{*}, \delta M^{*}\end{array}$ & $\begin{array}{c}(\alpha=-1.00) \\
M^{*}, \delta M^{*}\end{array}$ \\
\hline $\begin{array}{l}\text { DARC } \\
\text { Relaxed }\end{array}$ & $\begin{array}{l}-21.61,0.07 \\
-21.59,0.05\end{array}$ & $\begin{array}{l}-21.83,0.06 \\
-21.75,0.06\end{array}$ \\
\hline \multicolumn{3}{|c|}{ Red galaxies $(\alpha=-0.70)$} \\
\hline $\begin{array}{l}\text { DARC } \\
\text { Relaxed }\end{array}$ & $\begin{array}{l}-21.54,0.07 \\
-21.44,0.05\end{array}$ & $\begin{array}{l}-21.52,0.05 \\
-21.48,0.06\end{array}$ \\
\hline \multicolumn{3}{|c|}{ Blue galaxies $(\alpha=-2.00)$} \\
\hline $\begin{array}{l}\text { DARC } \\
\text { Relaxed }\end{array}$ & $\begin{array}{l}-22.74,0.52 \\
-22.38,0.11\end{array}$ & $\begin{array}{l}-23.18,0.11 \\
-23.56,0.06\end{array}$ \\
\hline
\end{tabular}

within $0.5 R_{200}$. However, this difference should be taken with care since the blue galaxy population is very poor in the internal regions of the clusters, which limits the number of considered counts.

\subsection{Butcher-Oemler effect}

The window opened by the redshift dependence of the galaxy properties has been used to set constraints on the time-scales of the processes of galaxy evolution (Butcher \& Oemler 1978, 1984; Stanford et al. 1998). In this context, the observational 


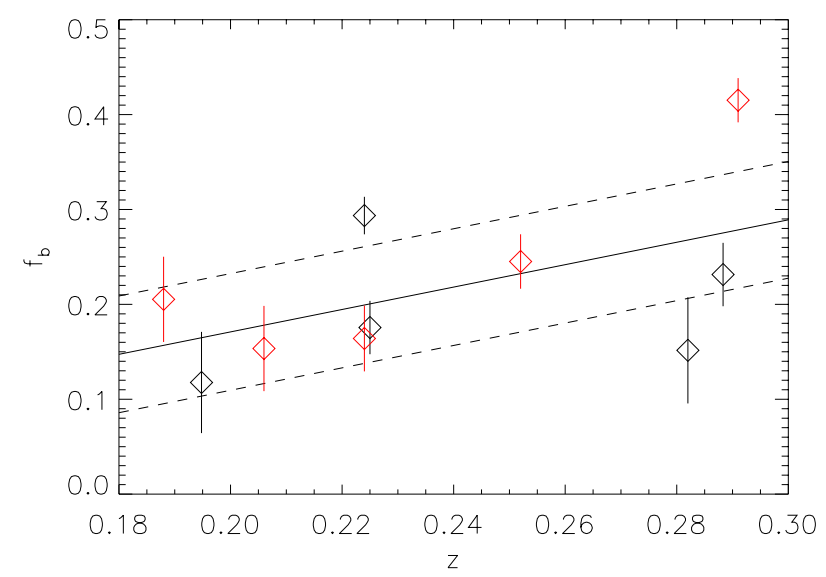

Fig. 10. Fraction of blue galaxies within $0.5 R_{200}$ for DARC (black symbols) and relaxed clusters (red symbols). The solid line describes the linear fit of this relation. The dashed lines show one-sigma uncertainties of the linear fit.

evidence of the environmental effect is still uncertain. To clarify whether merging processes in clusters can enhance the star formation in the galaxy populations, we compare the fraction of blue galaxies, $f_{\mathrm{B}}$, in DARC and relaxed clusters. To do this, we quantify the Butcher-Oemler effect (Butcher \& Oemler 1978, 1984) in two homogeneous cluster samples with similar velocity dispersions and masses.

The studies of Andreon et al. (2006) showed that the radial dependence of the blue fraction is quite shallow, and it smoothly and monotonically increases from the center of the cluster to the field (see Fig. 10 in Andreon et al. 2006). However, the contamination contribution of field galaxies is lower in the inner regions. For this reason, we estimate $f_{\mathrm{B}}$ within $0.5 R_{200}$.

Figure 10 shows that $f_{\mathrm{B}}$ increases toward high redshifts and is at the $95 \%$ c.l. according to the Kendall statistics. A linear fit gives $f_{\mathrm{B}}(z)=1.18( \pm 0.07) z-0.07( \pm 0.06)$ (see also Sect. 7 for more discussions). We find no significant difference between $f_{\mathrm{B}}$ of DARC and relaxed clusters.

\section{The bright end of the LF}

Here we define as bright galaxies (BGs) those galaxies with $M_{r^{\prime}}<-22.5$ and analyze whether DARC and relaxed clusters differ in their BGs, in counts or luminosity.

\subsection{Counting BGs}

Figure 11 shows the number of BGs for each cluster $N_{\mathrm{BG}}=$ $N_{r^{\prime}<-22.5} / N_{r^{\prime}<-20}\langle N\rangle$, where $N_{r^{\prime}<-22.5}$ is the number of galaxies brighter than $M_{r^{\prime}}<-22.5$ (normalized to the cluster richness, see Sect. 4) and $\langle N\rangle=\left\langle N_{r^{\prime}<-20}\right\rangle$ is the mean richness as computed from all clusters. Here we multiply by the mean richness to obtain more realistic BG counts. Error bars are assumed to be the Poissonian errors. The figure shows that the BG counts are higher in DARC clusters than in relaxed clusters. Considering the cluster regions within $R_{200}$, the mean values for both samples separately are $N_{\text {BG,DARC }}=23.1 \pm 3.1$ and $N_{\text {BG,rel }} 12.0 \pm 2.7$ for DARC and relaxed clusters. Therefore, DARC clusters contain a factor two more BGs than relaxed clusters, and the difference is significant at the $2.7 \sigma$ c.l.

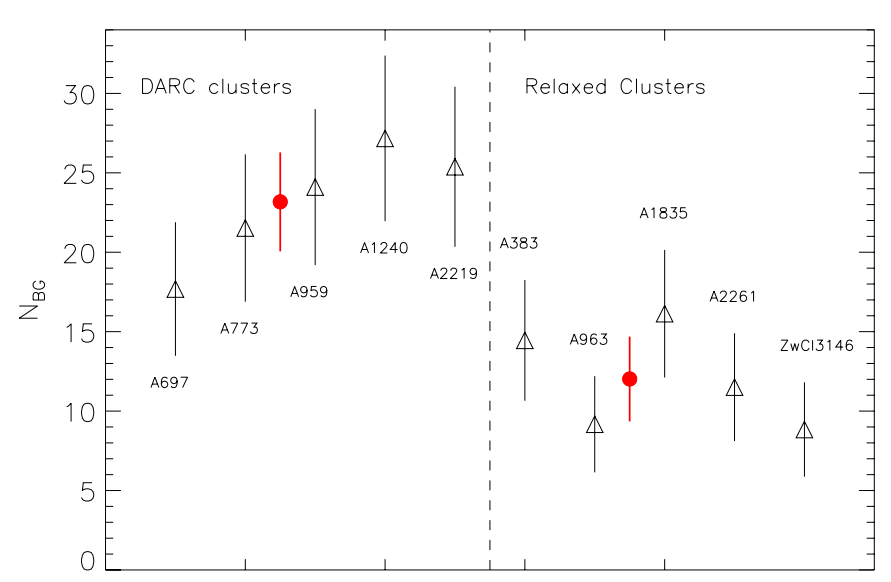

Fig. 11. Number of bright galaxies within $R_{200}$. Vertical dashed lines separate DARC and relaxed cluster samples. Labels indicate individual clusters. The red points represent the mean value computed for each cluster sample separately.
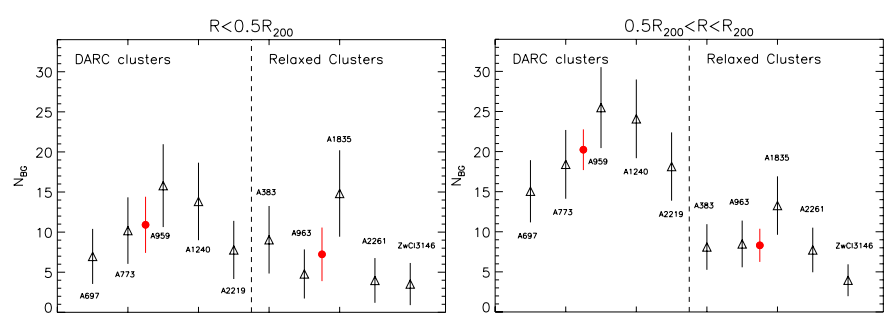

Fig. 12. BG counts in the inner region, $R<0.5 R_{200}$ (left panel) and in the outer region, $0.5 R_{200}<R<R_{200}$ (right panel). Symbols are the same as in Fig. 11.

We also studied the spatial distribution of BG galaxies. We computed BG counts in two separate regions, $R<0.5 R_{200}$ and $0.5 R_{200}<R<R_{200}$ (see Fig. 12). For the inner cluster region, $R<0.5 R_{200}$, we obtain $N_{\mathrm{BG}, \mathrm{DARC}}=10.9 \pm 2.7$ and $N_{\mathrm{BG}, \text { rel }}=$ $7.2 \pm 2.4$. For the outer cluster region, $0.5 R_{200}<R<R_{200}$, we obtain $N_{\text {BG,DARC }}=20.2 \pm 2.5$ and $N_{\text {BG,rel }}=8.3 \pm 2.1$. This shows that the difference in $\mathrm{BG}$ counts is restricted to the outer region, where it is significant at $3.6 \sigma$ c.l.

We correlated $N_{\mathrm{BG}}$ with the redshift and the mass of the cluster. We do not observe any clear relation in either the $N_{\mathrm{BG}}-z$ or the $N_{\mathrm{BG}}-M_{200}$ relations.

Our above results are based on photometric BG counts appropriately corrected with the average field counts (see Sect. 4.1). Owing to the poor number statistic of BGs in the individual clusters, we also used another approach to check the above results. We also resorted to the SDSS spectroscopic survey since redshift data can provide the correct cluster membership. Unfortunately, the SDSS survey is not spectroscopically complete. Indeed, within the cluster regions of our sample, SDSS DR8 provides redshifts $z$ for $60 \%$ of galaxies with $M_{r^{\prime}}<-22.5$. Therefore, we cannot assess the cluster membership for all BGs. Here we consider a mixed approach: we counted BGs in the photometric sample and then weighted these numbers on the base of the spectroscopic information.

Table 8 lists the mean number of BGs for DARC and relaxed cluster from photometric data, (Cols. 2 and 3), and the respective difference and ratio between $N_{\mathrm{BG}}$ in DARC and relaxed clusters (Cols. 4 and 5). These values confirm that the strong difference 
R. Barrena et al.: Environmental effects on the bright end of the galaxy luminosity function in galaxy clusters

Table 8. BGs in photometric samples weighted with spectroscopic samples.

\begin{tabular}{lrcccccc}
\hline \hline & \multicolumn{3}{c}{ From photometric survey } & \multicolumn{2}{c}{ From spectroscopic survey } & Photo+spec \\
& $N_{\text {BG,DARC }}$ & $N_{\text {BG,rel }}$ & Diff. & Ratio & $N_{\text {mem }} / N_{z}(\mathrm{DARC})^{a}$ & $N_{\text {mem }} / N_{z}(\text { rel })^{a}$ & WRatio $^{a}$ \\
\hline$R<R_{200}$ & 65 & 45 & $20 \pm 10$ & 1.4 & $10 / 41(8 / 41)$ & $8 / 25(6 / 25)$ & $1.1(0.9)$ \\
$R<0.5 R_{200}$ & 13 & 16 & $3 \pm 6$ & 0.8 & $4 / 13(4 / 13)$ & $4 / 8(4 / 8)$ & $0.5(0.5)$ \\
$R>0.5 R_{200}$ & 52 & 29 & $23 \pm 9$ & 1.8 & $6 / 28(4 / 28)$ & $4 / 17(2 / 17)$ & $1.6(2.2)$ \\
\hline
\end{tabular}

Notes. ${ }^{(a)}$ Where member galaxies are those with $\delta z=0.01(\delta z=0.007)$.

between DARC and relaxed clusters are due to the outer cluster region, while nothing can be said about the inner region because of the poor number statistics. Note that here we did not normalize with the respective cluster richness as in the above section: the net effect would have slightly reduced the observed difference between DARC and relaxed clusters. Therefore, the results of this section should be looked at as being very conservative.

We used the spectroscopic information to weight the above ratios. We considered as galaxy members those galaxies with a difference of $\delta z=0.01(\delta z=0.007)$ from the cluster redshift, i.e. $\delta c z \sim 3000 \mathrm{~km} \mathrm{~s}^{-1}\left(\sim 2000 \mathrm{~km} \mathrm{~s}^{-1}\right)$ from the mean cluster velocity. Table 8 lists the number of cluster BGs over the number of all BGs with redshift, $N_{\text {mem }} / N_{z}$, for DARC and relaxed cluster (Cols. 6 and 7), and the values of the ratios DARC/relaxed now weighted with the respective $N_{\text {mem }} / N_{z}$, i.e. WRatio(DARC/rel $)=$ Ratio $($ DARC $/$ rel $) *\left[N_{\text {mem }} / N_{z}(\right.$ DARC $\left.)\right] \times$ $\left[N_{\text {mem }} / N_{z}(\mathrm{rel})\right]^{-1}$ (Col. 8). When comparing the values of "WRatio" with those of above "Ratio" we confirm that DARC clusters are richer in BGs than relaxed cluster within $0.5 R_{200}$.

\section{BG luminosity}

We investigated whether the contribution in luminosity of BGs is different in DARC and relaxed clusters. We computed the "global" luminosity for $M_{\mathrm{r}^{\prime}}<-20$ using the counts of Sect. 4, i.e.

$L_{\text {global }}=\sum_{i}^{N} N_{i}(m) l_{i}(m)$,

where the sum is performed over all $N$ magnitude bins with galaxy number $N_{i}(m)$ and mean luminosity $l_{i}(m)$. The transformation from absolute magnitudes to absolute luminosity in units of solar luminosities is performed using the solar magnitude $M_{r \odot}=4.62$. The luminosity associated to BGs, $L_{\mathrm{BG}}$, is computed in the same way out to $M_{r^{\prime}}<-22.5$. Table 9 lists the luminosities for each cluster. Values within parenthesis are errors estimated at $1 \sigma$ c.l.

Note that $L_{\mathrm{BG}}$ represents a non-negligible fraction of the global luminosity for $M_{r^{\prime}}<-20$ and the ten clusters span a wide range of luminosities. We observe two dramatic cases, these are A1240 in the DARC cluster sample and A383 in the relaxed sample, where the luminosity fraction associated to the BGs, $f_{\mathrm{L}, \mathrm{BG}}$, is about $67 \%$ and $96 \%$ of the total luminosity, respectively. Considering the whole samples, we find that the mean luminosity fractions of BGs are $f_{\mathrm{L}, \mathrm{BG}, \mathrm{DARC}}=0.35 \pm 0.19$ and $f_{\mathrm{L}, \mathrm{BG}, \mathrm{rel}}=0.39 \pm 0.33$ for DARC and relaxed clusters. If we exclude the two extreme cases of A1240 and A383, we find $f_{\mathrm{L}, \mathrm{BG}, \mathrm{DARC}}=0.27 \pm 0.09$ and $f_{\mathrm{L}, \mathrm{BG} \text {,rel }}=0.25 \pm 0.13$. From these values, we conclude that DARC and relaxed clusters do not significantly differ for the luminosity content associated to their BGs.
Table 9. Luminosity content of BGs.

\begin{tabular}{lcc}
\hline \hline Name & $\begin{array}{c}L_{\text {global }} \\
\left(10^{12} L_{\odot}\right)\end{array}$ & $\begin{array}{c}L_{\mathrm{BG}} \\
\left(10^{12} L_{\odot}\right)\end{array}$ \\
\hline A697 & $4.38(0.24)$ & $0.68(0.04)$ \\
A773 & $10.71(2.33)$ & $3.43(0.75)$ \\
A959 & $7.20(2.11)$ & $2.53(0.74)$ \\
A1240 & $16.14(4.74)$ & $10.78(3.17)$ \\
A2219 & $12.08(2.96)$ & $3.32(0.81)$ \\
\hline A383 & $3.03(0.63)$ & $2.90(0.60)$ \\
A963 & $6.29(0.67)$ & $0.78(0.08)$ \\
A1835 & $16.95(2.74)$ & $7.04(1.14)$ \\
A2261 & $12.31(2.52)$ & $2.55(0.53)$ \\
ZwC13146 & $6.95(3.52)$ & $1.74(0.09)$ \\
\hline
\end{tabular}

\section{Discussion and conclusions}

We compared five unrelaxed clusters (DARC sample) with relaxed clusters. As far as possible, we contolled potential biases. The relaxed clusters are similar to unrelaxed clusters for their redshift range, X-ray temperature, and mass (see Tables 1 and 2). Moreover, we always worked within physical rather than fixed radii.

We found that very unrelaxed clusters are comparable to relaxed ones for the statistical properties, i.e. the parameters of the $\mathrm{LF}$ and the relative behaviour between red and blue galaxies. In particular, we agree with a series of previous results.

We compared $M^{*}$ and $\alpha$ parameters of the composite Schechter luminosity functions with results obtained by Popesso et al. (2006). Transforming their $M^{*}$ estimates (see their Tables 1 and 2) to our cosmology, applying the factor $5 \log \left(h_{70}\right)$, we obtained values that perfectly agree.

In addition, in agreement with Popesso et al. (2006, their Fig. 10) and de Filippis et al. (2011, their Fig. 11), the LF profile does not depend on the considered cluster region within $M_{r}<-19$.

We also found evidence of the variation of the blue fraction of galaxies with redshift. It is very remarkable that we can detect the effect even in a so limited redshift range. In particular, note that extrapolating our $f_{\mathrm{B}}(z)$ relation to $z=0.35$, we obtain a $f_{\mathrm{B}}(z=0.35)=0.34 \pm 0.05$. This estimate agrees well with those obtained by Andreon et al. (2006). They found $f_{\mathrm{B}}(z=0.35)=0.33 \pm 0.05$ with clusters located in a redshift range $0.3-0.4$. When extrapolating to $z=0.5$, we obtain $f_{\mathrm{B}}(z=0.5) \sim 0.5$. This finding agrees very well with cosmological galaxy formation models for clusters with similar mass (see Menci et al. 2008, their Fig. 3).

We found for the first time that relaxed clusters contain fewer BGs and these BGs are more concentrated in cluster inner regions. In contrast, unrelaxed clusters present more BGs that are more homogeneously distributed within the whole cluster. The $\mathrm{BG}$ richness of DARC clusters is the robust and significant result of Sect. 5. 
Our result does not contradict that of de Propris et al. (2003), who found a similar LF between substructured and non substructured clusters because: i) their study is devoted to LF parameters, for which we find no difference; ii) our sample of DARC clusters are likely to be much farther away from dynamical equilibrium than their sample of substructured clusters. Indeed, DARC clusters are found to be cases of major, very important mergers, in agreement with the rarity of the diffuse radio source phenomena ( $\sim 10 \%$ of clusters, Giovannini \& Feretti 2002). Moreover, DARC clusters should all be recent mergers since radio emissions are expected to have a short life time, i.e. of the order of a few $10^{8}$ years (e.g., Giovannini \& Feretti 2002; Skillman et al. 2010), in agreement with times estimated for a few individual clusters (e.g., Markevitch et al. 2002; Girardi et al. 2008).

We also found for the first time that the luminosity content in $\mathrm{BGs}$ is the same in DARC and relaxed clusters. The consequent scenario is that the (more numerous) BGs lying in the outer regions of merging clusters will merge to form (more luminous) BGs in the inner regions of relaxed clusters. This combined formation/evolution of BGs and the parent clusters agree well with the results of Lin \& Mohr (2004), who analyzed the correlation between BCG luminosity and parent cluster mass, supporting a recent formation of the brightest galaxies in the context of the hierarchical scenario.

We plan to extend our study to a larger sample of DARC clusters by examining their dynamics to compute more reliable mass estimates. Another possible extension of this work is to look at deeper magnitudes and to investigate possible effects on the faint end of the galaxy LF connected with the cluster evolution.

Acknowledgements. We are indebted to Andrea Biviano for his useful comments on this work. We are also grateful for the creation and distribution of the SDSS Archive, provided by the Alfred P. Sloan Foundation and other Participating Institutions. The SDSS Web site is http://www.sdss.org/. This work has been funded by the Spanish Ministry of Science and Innovation (MICINN) under the collaboration grants AYA2010-21887-C04-04 and AYA2010-21322-C03-02. MG acknowledges financial support from PRIN-INAF-2010.

\section{References}

Allen, S. W., Ettori, S., \& Fabian, A. C. 2001, MNRAS, 324, 877

Allen, S. W., Schmidt, R. W., Ebeling, H., Fabian, A. C., \& van Speybroeck, L. 2004, MNRAS, 353, 457

Andreon, S., Quintana, H., Tajer, M., Galaz, G., \& Surdej, J. 2006, MNRAS, 365,915

Ascaso, B., Aguerri, J. A. L., Varela, J., et al. 2011, ApJ, 726, 69

Baldi, A., Ettori, S., Tozzi, P., \& Borgani, S. 2007, ApJ, 666, 835

Barkhouse, W. A., Yee, H. K. C., \& López-Cruz, O. 2007, ApJ, 671, 1471

Barrena, R., Boschin, W., Girardi, M., \& Spolaor, M. 2007a, A\&A, 467, 37

Barrena, R., Girardi, M., Boschin, \& Dasí, M. 2009, A\&A, 503, 357

Bautz, L. P., \& Morgan, W. W. 1970, ApJ, 162, 149

Binggeli, B., Sandage, A., \& Tamman, G. A. 1988, ARA\&A, 26, 509

Biviano, A., Murante, G., Borgani, S., et al. 2006, A\&A, 456, 23

Boschin, W., Girardi, M., Barrena, R., et al. 2004, A\&A, 416, 839

Boschin, W., Barrena, R., Girardi, M., \& Spolaor, M. 2008, A\&A, 487, 33

Butcher, H., \& Oemler, A. 1978, ApJ, 219, 18

Brunetti, G., Cassano, R., Dolag, K., \& Setti, G. 2009, A\&A, 507, 661

Buote, D. A. 2002, in Merging Processes in Galaxy Clusters, ed. L. Feretti, I. M.

Gioia, \& G. Giovannini (The Netherlands: Kluwer Ac. Pub.)

Butcher, H., \& Oemler, A. 1984, ApJ, 285, 426

Carlberg, R. G., Yee, H. K. C., Ellingson, E., et al. 1996, ApJ, 462, 32

Carlberg, R. G., Yee, H. K. C., \& Ellingson, E. 1997, ApJ, 478, 462

Cassano, R., Ettori, S., Giacintucci, S., et al. 2010, ApJ, 721, 82

de Filippis, E., Paolillo, M., Longo, G., et al. 2011, MNRAS, 414, 277

de Lucia, G., \& Blaizot, J. 2007, MNRAS, 375, 2 de Propris, R., Pritchet, C. J., Harris, W. E., \& McClure, R. D. 1995, ApJ, 450, 534

de Propris., R., Colless, M., Driver, S. P., et al. 2003, MNRAS, 342, 725

Dressler, A. 1978, ApJ, 223, 765

Dressler, A. 1980, ApJ, 236, 351

Driver, S. P., Couch, W. J., \& Phillipps, S. 1998, MNRAS, 301, 369

Dubinski, J. 1998, ApJ, 502, 141

Durret, F., Laganá, T. F., \& Haider, M. 2011, A\&A, 529, A38

Ebeling, H., Voges, W., Böhringer, H., et al. 1996, MNRAS, 281, 799

Ebeling, H., Edge, A. C., Böhringer, H., et al. 1998, MNRAS, 301, 881

Ensslin, T. A., Biermann, P. L., Klein, U., \& Kohle, S. 1998, A\&A, 332, 395

Feretti, L. 2006, in Proc. of the XLIst Rencontres de Moriond, XXVIth Astrophysics Moriond Meeting: From dark halos to light, ed. L. Tresse, S.

Maurogordato, \& J. Tran Thanh Van [arXiv:astro-ph/0612185]

Feretti, L. 2008, Mem. SAIt, 79, 176

Feretti, L., Gioia I. M., \& Giovannini G. 2002, in Merging Processes in Galaxy Clusters (The Netherlands: Kluwer Academic Publisher), Astrophys. Space Sci. Lib., 272

Fukugita, M., Shimasaku, K., \& Ichikawa, T. 1995, PASP, 107, 945

Gilbank, D. G., Yee, H. K. C., Ellingson, E., et al. 2008, ApJ, 673, 742

Giovannini, G., \& Feretti, L. 2002, in Merging Processes in Galaxy Clusters, ed.

L. Feretti, I. M. Gioia, \& G. Giovannini (The Netherlands: Kluwer Ac. Pub.) Girardi, M., \& Mezzetti, M. 2001, ApJ, 548, 79

Girardi, M., Fadda, D., Giuricin, G., et al. 1996, ApJ, 457, 61

Girardi, M., Giuricin, G., Mardirossian, F., Mezzetti, M., \& Boschin, W. 1998, ApJ, 505, 74

Girardi, M., Boschin, W., \& Barrena, R. 2006, A\&A, 455, 45

Girardi, M., Barrena, R., \& Boschin, W. 2007, Contribution to Tracing Cosmic Evolution with Clusters of Galaxies: Six Years Later conference - http:// www.si.inaf.it/sesto2007/contributions/Girardi.pdf

Girardi, M., Barrena, R., Boschin, W., \& Ellingson, E. 2008, A\&A, 491, 379

Hilton, M., Collins, C., De Propris, R., et al. 2005, MNRAS, 363, 661

Iglesias-Páramo, J., Boselli, A., Gavazzi, G., Cortese, L., \& Vílchez, J. M. 2003, A\&A, 397, 421

Keshet, U. 2011, MNRAS, submitted [arXiv: 1011.0729]

Keshet, U., \& Loeb, A. 2010, ApJ, 722, 737

Lin, Y.-T., \& Mohr, J. J. 2004, ApJ, 617, 879

López-Cruz, O., Yee, H. K. C., Brown, J. P., Jones, C., \& Forman, W. 1997, ApJ, 475,97

Lu, T., Gilbank, D. G., Balogh, M. L., \& Bognat, A. 2009, MNRAS, 399, 1858

Markevitch, M., Gonzalez, A. H., David, L., et al. 2002, ApJ, 567, 27

Mastropietro, C., \& Burkert, A. 2008, MNRAS, 389, 967

Maughan, B. J., Jones, C., Forman, W., \& van Speybroeck, L. 2008, ApJS, 174, 117

Menci, N., Rosati, P., \& Gobat, R. 2008, ApJ, 685, 863

Mercurio, A., Massarotti, M., Merluzzi, P., La Barbera, F., \& Busarello, G. 2003, A\&A, 408, 57

Merritt, D. 1985, ApJ, 289, 18

Million, E. T., \& Allen, S. W. 2009, MNRAS, 399, 1307

Ostriker, J. P., \& Tremaine, S. D. 1975, ApJ, 202, 113

Pinkney, J., Roettiger, K., Burns, J. O., \& Bird, C. M. 1996, ApJS, 104, 1

Poggianti, B. M. 2005, Proc. Sci., ed. R. J. Dettmar, U. Klein, \& P. Salucci, Invited lecture at the Bochun/Bonn Graduate Research School Baryons in Dark Matter Halos, Novigrad, Oct. 2004, http://pos. sissa.it

Popesso, P., Böhringer, H., Brinkmann, J., Voges, W., \& York, D. G. 2004, A\&A, 423,449

Popesso, P., Biviano, A., Böhringer, H., \& Romaniello, M. 2006, A\&A, 445, 29

Ramella, M., Biviano, A., Pisani, A., et al. 2007, A\&A, 470, 39

Ricker, P. M., \& Sarazin, C. L. 2001, ApJ, 561, 621

Rines, K., \& Geller, M. J. 2008, AJ, 135, 1837

Roche, N., Bernardi, M., \& Hyde, J. 2009, MNRAS, 398, 1549

Rudnick, G., Von der Linden, A., Pelló, R., et al. 2009, ApJ, 700, 1559

Ruszkowski, M., \& Springel, V. 2009, ApJ, 696, 1094

Sanderson, A. J. R., Ponman, T. J., \& O'Sullivan, E. 2006, MNRAS, 372, 1496

Schechter, P. 1976, ApJ, 203, 297

Schlegel, D. J., Finkbeiner, D.P., \& Davis, M. 1998, ApJ, 500, 525

Skillman, S. W., Hallman, E. J., O'Shea, B. W., et al. 2011, ApJ, 735, 96

Stanford, S. A., Eisenhardt, P. R., \& Dickinson, M. 1998, ApJ, 492, 461

Stott, J. P., Collins, C. A., Sahlén, M., et al. 2010, ApJ, 718, 23

Tribble, P. C. 1993, MNRAS, 261, 57

Valotto, C. A., Nicotra, M. A., Muriel, H., \& Lambas, D. G. 1997, 479, 90

Valotto, C. A., Muriel, H., Moore, B., \& Lambas, D. G. 2004, 601, 67

Whitmore, B. C., \& Gilmore, D. M. 1991, ApJ, 367, 64 